\title{
EXTENDABILITY OF SOLUTIONS OF HELMHOLTZ'S EQUATION TO THE INTERIOR OF A TWO-DIMENSIONAL SCATTERER
}

\author{
BY \\ RAUL KANGRO (Dept. of Mathematics, Tartu University, Tartu, Estonia), \\ URVE KANGRO (Dept. of Mathematics, Tartu University, Tartu, Estonia),
}

AND

ROY NICOLAIDES (Dept. of Mathematical Sciences, Carnegie Mellon University, Pittsburgh, Pennsylvania)

Introduction. This paper discusses the question of extending solutions of the twodimensional Helmholtz equation across a curve. Although general results on extending solutions of elliptic systems are available [6], these results do not normally give useful information about the size of an extension. In most applications though, the size of the extension region matters. Our main result, stated in the following section, gives explicit criteria which can be used to determine a region in which an extension is guaranteed to exist. The criteria are easy to check and, often, the region that they determine is maximal in a natural sense.

A classical application of extension results for the Helmholtz equation is to the socalled Rayleigh hypothesis. In 1907 Rayleigh solved the two-dimensional scattering problem for a sinusoidal surface illuminated by a plane wave in terms of a series of outgoing waves [8]. Assuming the validity of the expansion for points above the peaks of the sinusoid, there remains the question about whether it gives the correct results in the troughs of the surface. To resolve this issue it is necessary to extend the expansion to the region between the peaks and troughs of the scatterer. It was eventually shown that for a sinusoida! surface there is a relation between the amplitude and period of the surface in order for an extension to exist. Rayleigh's solution is correct only under this constraint on the surface geometry. A discussion may be found in [1].

Subsequently, numerous attempts were made to obtain similar results without using an explicit formula for the solution. The best known results were obtained by Millar [5]. This paper represents the solution of the Helmholtz equation using a form of Green's theorem in the complex plane and applies conformal mapping arguments based on the Schwartz function to locate the singularities. It seems that this approach may require

Received May 18, 1998.

2000 Mathematics Subject Classification. Primary 35J05, 65N12, 35Q60.

The work of the third author was supported by the United States Air Force Office of Scientific Research under Grant F49620-97-1-0204. 
prior constraints on the type of singularities that can be located [5, p. 160]. On the other hand, it is independent of parameterizations of the curve, which our approach is not.

Our interest in extending solutions of Helmholtz's equation arose from a realization that these extensions control the rate of convergence of a numerical method for solving scattering problems. In this method, an approximate solution is sought as a linear combination of fundamental solutions satisfying the radiation condition. The poles of the fundamental solutions are located on a contour strictly inside the scatterer. The coefficients of the approximation are determined by collocation or least squares matching of the incident field on the boundary of the scatterer. Such an approximation automatically provides an approximate (and analytic) extension of the exact solution inside the scatterer but outside the contour. Naturally, the algorithm will work best when the exact extension to this region is free of singularities (see, however, [2]). The effect of singularities of the extension on the behavior of these "interior source" algorithms is explained more fully in another forthcoming paper [4]. A general survey of interior source methods in acoustics may be found in [7].

In this paper we use a fresh approach to obtain the extension. It is based on a transformation of coordinates followed by a transformation of the governing elliptic equation to a hyperbolic equation using the ideas of complex characteristics [3, Chapter 16]. The hyperbolic equation is solved to make the desired extension. Although this technique is not inherently two dimensional, in more than two dimensions the computations become quite involved. Because of this we deal only with the two-dimensional case, and the paper is organized as follows: the following section states the hypotheses for the extension theorem. These hypotheses are the "explicit criteria" referred to above. Then, Sec. 3 contains the proof of the extension theorem with some technical results deferred to Sec. 4. Section 5 contains an example that is designed to illustrate the use of the theorem in a typical situation. We have omitted the application to the classical Rayleigh problem since it is relatively simple and yields only the known result.

1. Assumptions. Let $\Omega$ be an open region in $\mathbf{R}^{2}$. Under assumptions (A1)-(A4) below, we will obtain an extension of a given solution of the Helmholtz equation in $\Omega$ to a larger region as a solution of the same equation. The first assumption is that a function $u$ satisfies

$$
u \in C^{2}(\bar{\Omega}), \quad \Delta u+\lambda u=0 \quad \text { in } \Omega,
$$

where $\lambda$ is a real or complex constant. Next, we isolate a part $\Gamma$ of the boundary $\partial \Omega$, about which we assume

$\Gamma \subset \partial \Omega, \Gamma=\{(f(t), g(t)), a \leq t \leq b\}$, where $f$ and $g$ are real analytic on $[a, b]$ with their complex analytic extensions $f(t+i \tau), g(t+i \tau)$ defined for $(t, \tau) \in[a, b] \times[-c, c]$.

Recall that all eigenfunctions of the Laplacian are real analytic in the interior of their domain of definition. Then, in order for $u$ to be extendable across $\Gamma$, the restriction of $u$ 
to $\Gamma$ must be a real analytic function. This motivates the third assumption.

The function $u_{\Gamma}$ defined by $u_{\Gamma}(t)=u(f(t), g(t)), t \in[a, b]$ is real analytic with its complex analytic extension $u_{\Gamma}(t+i \tau)$ defined for $(t, \tau) \in[a, b] \times[-c, c]$.

It follows from the proof of Theorem 1 below that the assumptions (A1)-(A3) are enough to ensure the existence of an extension of $u$ as a solution to the Helmholtz equation to a region containing the interior of $\Gamma$. To show how far across $\Gamma$ the extension is guaranteed to exist we introduce a mapping $\Psi$ defined by

$$
\Psi(t, \tau)=(\operatorname{Re}(F(t+i \tau)), \operatorname{Im}(F(t+i \tau))),
$$

where $F(t)=f(t)+i g(t)$. The fourth and the last assumption is then

$$
\begin{gathered}
\Psi([a, b] \times(0, c)) \in \Omega, \quad \Psi([a, b] \times(-c, 0)) \in \mathbf{R}^{2} \backslash \Omega, \\
|\operatorname{det}(\nabla \Psi(t, \tau))|=\left|F^{\prime}(t+i \tau)\right|^{2} \leq C<\infty \quad \forall(t, \tau) \in[a, b] \times[-c, c],
\end{gathered}
$$

$\Psi$ is injective in $[a, b] \times[-c, 0]$.

Note that a necessary condition for (A4) to hold is

$$
|\operatorname{det}(\nabla \Psi(t, \tau))|>0 \quad \forall(t, \tau) \in(a, b) \times(-c, 0] .
$$

2. Extension Theorem. The extension theorem is as follows:

TheOREM 1. Under the assumptions (A1)-(A4), there exists $\tilde{u} \in C^{2}(\bar{\Omega} \cup \Psi([a, b] \times[-c, 0])$ satisfying $\tilde{u}=u$ on $\bar{\Omega}$ and

$$
\Delta \tilde{u}+\lambda \tilde{u}=0 \quad \text { on } \Omega \cup \Psi((a, b) \times(-c, 0]) .
$$

Proof. First we use $\Psi$ to flatten the boundary curve $\Gamma$. Then, by complexifying the resulting equation with respect to one variable, we reduce the question of extension to finding sufficiently regular solutions to two hyperbolic equations.

Define $v \in C^{2}([a, b] \times[0, c])$ by $v=u \circ \Psi$. Then

$$
\Delta v=\Delta \Psi \cdot \nabla u \circ \Psi+\nabla \Psi \nabla \Psi^{\mathrm{T}}: \nabla^{2} u \circ \Psi .
$$

For $\Psi$ we have $\Delta \Psi=0$ and $\nabla \Psi \nabla \Psi^{\mathrm{T}}=\operatorname{det}(\nabla \Psi) I$. Thus $v$ satisfies

$$
\nabla v+h v=0 \quad \text { in }(a, b) \times(0, c),
$$

where $h(t, \tau)=\lambda \operatorname{det}(\nabla \Psi(t, \tau))=\lambda\left|F^{\prime}(t+i \tau)\right|^{2}$. We shall construct an extension of $u$ by extending $v$ to $(a, b) \times(-c, c)$ as a solution of $\Delta \tilde{v}+h \tilde{v}=0$, and then defining

$$
\tilde{u}(x)= \begin{cases}u(x), & x \in \Omega, \\ \tilde{v}\left(\Psi^{-1}(x)\right), & x \in \Psi([a, b] \times[-c, 0]) .\end{cases}
$$

Here (A4) guarantees the existence of $\Psi^{-1}$.

The method is motivated by the following observations. First, if $\tilde{v}$ is the extension of $v$, then its complex analytic extension with respect to the $t$ variable $(\tilde{v}(t+i \eta, \tau))$ formally satisfies the wave equation

$$
\left(-\frac{\partial^{2}}{\partial \eta^{2}}+\frac{\partial^{2}}{\partial \tau^{2}}\right) \tilde{v}(t+i \eta, \tau)+h(t+i \eta, \tau) \tilde{v}(t+i \eta, \tau)=0
$$


Conversely, if a $C^{2}$-function $\widetilde{V}$ satisfies

$$
\begin{gathered}
\left(-\frac{\partial^{2}}{\partial \eta^{2}}+\frac{\partial^{2}}{\partial \tau^{2}}\right) \tilde{V}(t, \eta, \tau)+h(t+i \eta, \tau) \tilde{V}(t, \eta, \tau)=0 \\
\left(\frac{\partial}{\partial t}+i \frac{\partial}{\partial \eta}\right) \tilde{V}(t, \eta, \tau)=0
\end{gathered}
$$

and $\widetilde{V}(t, 0, \tau)=v(t, \tau),(t, \tau) \in[a, b] \times[0, c]$, then $\widetilde{V}(t, 0, \tau)$ is the desired extension of $v$ (since it satisfies $\left.\left(\frac{\partial^{2}}{\partial t^{2}}+\frac{\partial^{2}}{\partial \tau^{2}}\right) \tilde{V}(t, 0, \tau)+h(t, \tau) \tilde{V}(t, 0, \tau)=0\right)$.

We prove the existence of $\tilde{V}$ in two steps, first for $\tau \geq 0$ and then for $\tau<0$.

I. Denote by $H$ the complex analytic extension of $h$ with respect to its first variable, i.e.,

$$
H(t, \eta ; \tau)=h(t+i \eta, \tau)=\lambda F^{\prime}(t+i(\tau+\eta)) \overline{F^{\prime}(t+i(\tau-\eta))}
$$

Note that $H$ is real analytic in $\{(t, \eta, \tau)|(t, \tau) \in[a, b] \times[-c, c],| \eta|\leq c-| \tau \mid\}$.

Consider the problem

$$
\left\{\begin{array}{l}
V_{\eta \eta}-V_{\tau \tau}=H V, \quad(t, \tau) \in(a, b) \times(0, c), \tau-c<\eta<c-\tau \\
V(t, 0 ; \tau)=v(t, \tau), \quad V_{\eta}(t, 0 ; \tau)=i v_{t}(t, \tau), \quad(t, \tau) \in[a, b] \times[0, c], \\
V(t, \eta ; 0)=u_{\Gamma}(t+i \eta), \quad a \leq t \leq b,-c \leq \eta \leq c
\end{array}\right.
$$

The following regularity result holds.

Lemma 1. Assume (A1)-(A3). Then the problem (3) has a solution of class $C^{2}$ in

$$
R^{+}=\{(t, \eta ; \tau) \mid a \leq t \leq b, 0 \leq \tau \leq c, \tau-c \leq \eta \leq c-\tau\}
$$

satisfying $\left(\frac{\partial}{\partial t}+i \frac{\partial}{\partial \eta}\right) V=0$ in $R^{+}$.

We prove the lemma in the next section.

Let $V$ be a $C^{2}$-solution of (3) satisfying $\left(\frac{\partial}{\partial t}+i \frac{\partial}{\partial \eta}\right) V=0$ in $R^{+}$. Then, in particular,

$$
V_{\tau}(\cdot, \cdot ; 0) \in C^{2}([a, b] \times[-c, c]), \quad\left(\frac{\partial}{\partial t}+i \frac{\partial}{\partial \eta}\right) V_{\tau}(t, \eta ; 0)=0 .
$$

II. Next we extend $V$ to the region

$$
R^{-}=\{(t, \eta ; \tau) \mid a \leq t \leq b,-c \leq \tau \leq 0,-\tau-c \leq \eta \leq c+\tau\}
$$

We use the following result that will be proved in the next section.

LEMMA 2. The initial value problem

$$
\left\{\begin{array}{l}
\widetilde{V}_{\tau \tau}-\widetilde{V}_{\eta \eta}+H \widetilde{V}=0 \quad \text { in } R^{-}, \\
\widetilde{V}(t, \eta ; 0)=v(t+i \eta, 0), \quad \widetilde{V}_{\tau}(t, \eta, 0)=V_{\tau}(t, \eta, 0), \quad(t, \eta) \in(a, b) \times(-c, c)
\end{array}\right.
$$

has a $C^{2}$-solution satisfying

$$
\widetilde{V}_{t}+i \widetilde{V}_{\eta}=0, \quad(t, \tau) \in[a, b] \times[-c, 0], \quad|\eta|<c+\tau .
$$


Let $\widetilde{V}$ be as in Lemma 2. Then, in particular, $\widetilde{V}$ satisfies $\widetilde{V}_{t t}+\widetilde{V}_{\eta \eta}=0$. This together with the hyperbolic equation above implies that $\tilde{v}(t, \tau)=\widetilde{V}(t, 0 ; \tau),(t, \tau) \in(a, b) \times(-c, 0)$ satisfies

$$
\Delta \tilde{v}+h \tilde{v}=0 \quad \text { in }(a, b) \times(-c, 0)
$$

with boundary conditions $\tilde{v}(t, 0)=v(t, 0), \tilde{v}_{\tau}(t, 0)=v_{\tau}(t, 0)$. Hence $\tilde{v}$ defined by

$$
\tilde{v}(t, \tau)= \begin{cases}v(t, \tau) & \text { if } \tau \geq 0 \\ \tilde{V}(t, 0 ; \tau) & \text { if } \tau<0\end{cases}
$$

satisfies

$$
\Delta \tilde{v}+h \tilde{v}=0 \quad \text { in }(a, b) \times(-c, c)
$$

This proves the theorem.

\section{Proofs of Lemmas.}

3.1. Proof of Lemma 1. We first reduce (3) to a Cauchy problem without any boundary conditions.

Define

$$
q(t, \eta ; \tau)=u_{\Gamma}(t+i \tau)-\frac{1}{2} \tau^{2}\left(u_{\Gamma}^{\prime \prime}(t+i \eta)+H(t, \eta ; 0) u_{\Gamma}(t+i \eta)\right)
$$

and let $W$ denote the odd extension of $V-q$ across the plane $\tau=0$ :

$$
W= \begin{cases}V(t, \eta ; \tau)-q(t, \eta ; \tau) & \text { if } \tau \geq 0 \\ q(t, \eta ;-\tau)-V(t, \eta ;-\tau) & \text { if } \tau<0\end{cases}
$$

Notice that if $V$ is a solution of (3), then $W$ satisfies

$$
\left\{\begin{array}{l}
W_{\eta \eta}-W_{\tau \tau}=H^{\mathrm{ev}} W-Q^{\text {od }} \quad \text { in } R \\
W(t, 0 ; \tau)=w^{\text {od }}(t, \tau), \quad W_{\eta}(t, 0 ; \tau)=i w_{t}^{\text {od }}(t, \tau), \quad a \leq t \leq b,|\tau| \leq c
\end{array}\right.
$$

where

$$
\begin{gathered}
R=\{(t, \eta ; \tau) \mid a \leq t \leq b,-c \leq \tau \leq c,-\tau-c \leq \eta \leq c+\tau\} \\
Q(t, \eta ; \tau)=q_{\eta \eta}-q_{\tau \tau}-H q \\
w(t, \tau)=v(t, \tau)-q(t, 0 ; \tau)
\end{gathered}
$$

and the superscripts od and ev denote odd and even extensions of a function from the region $\tau \geq 0$ into $\tau<0$, respectively. Note that $Q$ is smooth in $\Omega$ and satisfies $Q(t, \eta ; 0)=$ $0, a \leq t \leq b,|\eta| \leq c$, and that $w(t, 0)=w_{\tau \tau}(t, 0)=0$. This guarantees sufficient smoothness of the corresponding extensions. 
We can construct a solution of (5) by an iteration method. Define $W^{0}(t, \eta ; \tau)=0$ and

$$
\begin{aligned}
& W^{n+1}(t, \eta ; \tau)=\frac{1}{2} w^{\text {od }}(t, \tau-\eta)+\frac{1}{2} w^{\text {od }}(t, \tau+\eta)+\frac{i}{2} \int_{\tau-\eta}^{\tau+\eta} w_{t}^{\text {od }}(t, s) d s \\
&+\frac{1}{2} \int_{0}^{\eta} \int_{\tau-\eta+\alpha}^{\tau+\eta-\alpha}\left(H^{\mathrm{ev}}(t, \alpha ; \beta) W^{n}(t, \alpha ; \beta)-Q^{\mathrm{od}}(t, \alpha ; \beta)\right) d \beta d \alpha, \\
& \quad(t, \tau) \in[a, b] \times[-c, c], \quad|\tau|-c \leq \eta \leq c-|\tau|, \quad n=0,1, \ldots .
\end{aligned}
$$

Then $W^{n}, n=0,1, \ldots$ are odd functions with respect to $\tau$. Using the equality

$$
\begin{aligned}
& W^{n+1}(t, \eta ; \tau)-W^{n}(t, \eta ; \tau) \\
& \quad=\frac{1}{2} \int_{0}^{\eta} \int_{\tau-\eta+\alpha}^{\tau+\eta-\alpha} H^{\mathrm{ev}}(t, \alpha ; \beta)\left(W^{n}(t, \alpha ; \beta)-W^{n-1}(t, \alpha ; \beta)\right) d \beta d \alpha
\end{aligned}
$$

and mathematical induction (starting with lower-order derivatives), we get

$$
\left.\| D^{\gamma}\left(W^{n+1}-W^{n}\right)\right) \|_{C(R)} \leq \frac{K C^{n} \eta^{2 n-|\gamma|}}{(2 n-|\gamma|) !}, \quad n=1,2, \ldots,
$$

where

$$
\gamma=\left(\gamma_{1}, \gamma_{2}, \gamma_{3}\right), \quad|\gamma|=\gamma_{1}+\gamma_{2}+\gamma_{3} \leq 2, \quad D^{\gamma}=\frac{\partial^{\gamma_{1}}}{\partial t^{\gamma_{1}}} \frac{\partial^{\gamma_{2}}}{\partial \eta^{\gamma_{2}}} \frac{\partial^{\gamma_{3}}}{\partial \tau^{\gamma_{3}}}
$$

and the constants $K, C$ depend on

$$
\left\|w^{\mathrm{od}}\right\|_{C^{2}([a, b] \times[-c, c])}, \quad\left\|Q^{\mathrm{od}}\right\|_{W^{1, \infty}(R)}, \quad\left\|H^{\mathrm{ev}}\right\|_{W^{1, \infty}(R)}
$$

and the maximum of $\left|Q_{t t}^{\text {od }}\right|,\left|H_{t t}^{\text {ev }}\right|$ in $R$. Hence $\left(W^{n}\right)_{n=0,1, \ldots}$ converges to $W$ in $C^{2}(R)$. Moreover, since

$$
\begin{aligned}
\left(\frac{\partial}{\partial t}+\right. & \left.i \frac{\partial}{\partial \eta}\right) W^{n+1}(t, \eta ; \tau) \\
= & \frac{i}{2} \int_{\tau-\eta}^{\tau+\eta}\left(w_{t t}^{\mathrm{od}}(t, s)+w_{\tau \tau}^{\mathrm{od}}(t, s)+H^{\mathrm{ev}}(t, 0 ; s) w^{\mathrm{od}}(t, s)+Q^{\mathrm{od}}(t, 0 ; s)\right) d s \\
& +\frac{1}{2} \int_{0}^{\eta} \int_{\tau-\eta+\alpha}^{\tau+\eta-\alpha}\left(\frac{\partial}{\partial t}+i \frac{\partial}{\partial \alpha}\right)\left(\left(H^{\mathrm{ev}} W^{n}-Q^{\mathrm{od}}\right)(t, \alpha ; \beta)\right) d \beta d \alpha \\
= & \frac{1}{2} \int_{0}^{\eta} \int_{\tau-\eta+\alpha}^{\tau+\eta-\alpha} H^{\mathrm{ev}}(t, \alpha ; \beta)\left(\frac{\partial}{\partial t}+i \frac{\partial}{\partial \alpha}\right)\left(W^{n}(t, \alpha ; \beta) d \beta d \alpha\right.
\end{aligned}
$$

we have

$$
\left(\frac{\partial}{\partial t}+i \frac{\partial}{\partial \eta}\right) W^{n}(t, \eta ; \tau)=0 \quad \text { in } R, n=0,1, \ldots
$$

and therefore $\left(\frac{\partial}{\partial t}+i \frac{\partial}{\partial \eta}\right) W(t, \eta ; \tau)=0$ in $R$. This proves that $V=W+q$ is a $C^{2}$-solution of (3) satisfying $\left(\frac{\partial}{\partial t}+i \frac{\partial}{\partial \eta}\right) V(t, \eta ; \tau)=0$ in $R^{+}$. 
3.2. Proof of Lemma 2. Define $\widetilde{V}^{0}=0$ and

$$
\begin{aligned}
\tilde{V}^{n+1}=\frac{1}{2} & \left(v(t+i(\eta-\tau), 0)+v(t+i(\eta+\tau), 0)+\int_{\eta-\tau}^{\eta+\tau} V_{\tau}(t, s) d s\right) \\
& -\frac{1}{2} \int_{0}^{\tau} \int_{\eta-\tau+\alpha}^{\eta+\tau-\alpha} H(t, \beta ; \alpha) \widetilde{V}^{n}(t, \beta ; \alpha) d \beta d \alpha, \quad n=0,1, \ldots
\end{aligned}
$$

As in the proof of Lemma 1 it now follows that $\tilde{V}^{n}$ is convergent in $C^{2}\left(R^{-}\right)$and that the limit $\widetilde{V}$ satisfies

$$
\widetilde{V}_{t}+i \widetilde{V}_{\eta}=0 \text { in } R^{-} .
$$

One can check by direct computation that $\widetilde{V}$ solves the Cauchy problem (4).

4. Location of the Singularities of the Extension. In this section we discuss how to use Theorem 1 to locate the singularities of the extension of a solution of the Helmholtz equation in some region $\Omega$ with boundary $\Gamma$. First one has to find an analytic mapping $F$ that maps a segment of the real axis to a piece of the boundary of $\Omega$. Note that any analytic parameterization of the boundary creates one such mapping. We identify $\mathbf{R}^{2}$ with the complex plane $\mathbf{C}$, extend $F$ to a neighborhood of the real axis and assume (as in A4) that complex numbers with positive imaginary parts map into $\Omega$. We can use several different mappings to cover $\Gamma$ but their images must overlap on some open subsets of $\Gamma$ to guarantee the compatibility of the extensions across the pieces of the boundary corresponding to different mappings. Even when it is easy to describe $\Gamma$ with one mapping, using the extension theorem with different $c$ for different boundary parts may give a larger region where the extension is guaranteed to exist.

There are several possible reasons for the appearance of singularities in the extension:

1. Singularities of $F$. If $|F(z)|=\infty, \operatorname{Im} z>0$, then the extension may have a singularity at the point $F(\bar{z})$. If $F$ has a branch point at $z$, then if $\operatorname{Im} z>0$, it may create a branch point of the extension at $F(\bar{z})$, and if $\operatorname{Im} z<0$ there may be a region (a curvilinear sector with the vertex at $F(z)$ ), where the extension cannot be defined in a straightforward way (although using a different mapping may help).

2. Singularities of the inverse function $F^{-1}$. These are the points where $F^{\prime}(z)=0$. Only points with $\operatorname{Im} z<0$ can create singularities of the extension, and they lie at the points $F(z)$; they are branch points (if the root of the derivative is simple, then the extension behaves like a square root near the singularity).

3. There may be a singularity (or singularities) at $F(t-i \infty), t \in \mathbf{R}$. This happens, for example, when extending the solution from the exterior of a circle to the interior.

4. Singularities of the complex analytic extension of the boundary value may aiso generate singularities of the extension of the solution. If the boundary value is a restriction to the boundary of a function, satisfying the Helmholtz equation on the whole plane, then the singularities of the extension of $u_{\Gamma}$ can be only at the points where $F$ is singular; so they can generate singularities of the extension of the solution only at points already discussed in 1 . If the boundary value is generated by a function, satisfying the Helmholtz equation everywhere except at one point $F(z)$, it may generate a singularity of the extension at the point $F(\bar{z})$ if $\operatorname{Im} z>0$ or at the point $F(z)$ if $\operatorname{Im} z<0$. 
5. The extension theorem does not give any information about extending the solution into the parts of $\mathbf{C} \backslash \Omega$ that are not in the image of the lower half-plane under the mapping $F$. In this case using a different mapping may help to obtain information. Also, if there are points with $\operatorname{Im} z>0$ and $F(z) \in \mathbf{C} \backslash \Omega$, an additional investigation will be needed about what happens at points $F(\bar{z})$. Of course, this means that (A4) is not satisfied in a strip wide enough to include $z$ and $\bar{z}$, but it may be possible to extend the solution to the points $F(z) \in \mathbf{C} \backslash \Omega, \operatorname{Im} z>0$ if they are in the image of the lower half-plane under the mapping $F$, and then use these values of the solution to define the extension at $F(\bar{z})$.

Since our extension theorem is local, it is also possible to use it for piecewise analytic boundaries. In this case the extensions across the different parts of the boundary are not compatible in general; at the points where the boundary is not analytic we may have branch points of the extension or curvilinear sectors where the extension is not defined.

An example. Consider the generalized ellipse, given by the equation

$$
\left(\frac{x}{a}\right)^{p}+\left(\frac{y}{b}\right)^{p}=1
$$

where $p$ is an even natural number and $a, b>0$. Our goal is to find the singularities of the analytic extension of a solution of the exterior Dirichlet problem for the Helmholtz equation with analytic boundary data. Using elliptic coordinates to parameterize the boundary, we find the mapping

$$
F(z)=\frac{a \cos z-i b \sin z}{\left((\cos z)^{p}+(\sin z)^{p}\right)^{1 / p}},
$$

which maps the real axis $2 \pi$-periodically onto the generalized ellipse. The next step is to check the possibilities for singularities.

1. The mapping has a singularity only at the points where the denominator is zero, i.e., when $(\tan z)^{p}=-1$. Since $\tan \bar{z}=\overline{\tan z}$, we see that for these points $|F(\bar{z})|=\infty$; so they do not generate any singularities of the extension. On the $z$-plane these points lie on the lines $\operatorname{Re} z= \pm \frac{\pi}{4}+k \pi, k \in \mathbf{Z}$ and so we have to cut the plane along these lines from points lying closest to the real axis to infinity.

2. Find the points where $F^{\prime}(z)=0$. Solving the equation $F^{\prime}(z)=0$ we get

$$
\tan z= \pm i \quad \text { or } \quad \tan z=\left(\frac{b}{a}\right)^{\frac{1}{p-1}} e^{\frac{(4 k-1) \pi i}{2(p-1)}}, k=1, \ldots, p-1
$$

Since $\operatorname{Im} \tan z<0$ if and only if $\operatorname{Im} z<0$, we have to consider only the points for which

$$
\tan z=-i \quad \text { or } \quad \tan z=\left(\frac{b}{a}\right)^{\frac{1}{p-1}} e^{\frac{(4 k-1) \pi i}{2(p-1)}}, k=\frac{p}{2}, \ldots, p-1 .
$$

The first one of these corresponds to $z=\xi-i \infty, \xi \in \mathbf{R}$; we will consider this point below. To find where the singularities caused by the others are, we have to be careful which branch of the $p$-th root to choose when calculating $F(z)$. The branch is determined by the requirement that the function has to be analytic on lines parallel to the imaginary axis (except the ones corresponding to branch cuts). 
Assuming for definiteness that $a>b$, we get that the singularities of the extension lie at the points

$$
\pm\left(a^{\frac{p}{p-1}}-i b^{\frac{p}{p-1}} e^{\frac{(4 k-1) \pi i}{2(p-1)}}\right)^{\frac{p-1}{p}}, \quad k=\frac{p}{2}, \ldots, p-1,
$$

where the branch cut for the root is along the negative real axis as usual (the expression in the parentheses has always positive real part). For $p$ large, these points lie roughly on semicircles with diameters on the shorter side of the generalized ellipse (which approximates a rectangle).

If $a=b$ all zeros of $F^{\prime}(z)$ lie on lines $\operatorname{Re} z= \pm \frac{\pi}{4}+k \pi, k \in \mathbf{Z}$. Apart from those nearest to the real axis they lie on the branch cuts; so it is possible to approach them from both sides. Hence the singularities of the extension lie at the points

$$
\pm a\left(1-i e^{\frac{(4 k-1) \pi i}{2(p-1)}}\right)^{\frac{p-1}{p}}, \quad k=\frac{p}{2}, \ldots, p-1
$$

and

$$
\pm a\left(e^{\frac{(4 k-1) \pi i}{2(p-1)}}-i\right)^{\frac{p-1}{p}}, \quad k=0, \ldots, \frac{p}{2}-1
$$

Some of these points coincide (those nearest to the "corners" of the body, and if $p$ is not divisible by 4 , then also the origin). They lie roughly on semicircles with diameters on both sides of the generalized ellipse.

3. To check the point $F(\xi-i \infty)$ one has to use a different mapping. If $p$ is not divisible by 4 , then $|F(\xi-i \infty)|=\infty$; so this does not create a singularity of the extension. If $p=4 n, n \in \mathbf{N}$, using $\zeta= \pm \tan z$ and $\zeta= \pm \cot z$ as new parameters, we get 4 different mappings that cover the boundary. Using these it is easy to show that the extension does not have a singularity at the point $F(\xi-i \infty)$.

4. In this example we will ignore the singularities that may be caused by the boundary data (assuming that the boundary value is regular enough).

5. The easiest way to check that the image of the lower half of the complex plane covers all of $\mathbf{C} \backslash \Omega$ is to draw the image (or a part of it) using mathematical software (we used Maple). Then one can see that, indeed, the whole interior of the generalized ellipse is covered and parts of it are covered more than once. This means that the extension into these regions is multiple-valued with the value depending on which part of the boundary one started. To get a single-valued extension, branch cuts must be inserted inside the multiply covered regions.

If $a=b$ the most natural place for the branch cuts is along the "diagonals", starting from the singularities nearest to the "corners" of the body. Note that in this case the extension of the solution is analytic up to the branch cut, except for the singularities near the "corners" and possibly at the origin (if $p$ is not divisible by 4 ). The other singularities of the extension are unreachable without crossing the branch cut.

If $a>b$, then a natural shape for the branch cut is $><$ where there is some freedom about choosing the length of the middle part. Again, the extension of the solution is analytic up to the branch cut, except for the singularities near the "corners" and possibly two singularities on the $x$-axis (if $p$ is not divisible by 4 ). 
5. Conclusions. We have shown how solutions of Helmholtz's equations can be extended across curves in the plane. The value of our approach is that it provides a prescription for actually computing a region (generally speaking, maximal) in which the extension exists. The usual approaches show that an extension exists but do not normally supply its domain of existence even though in applications the size of the extension can be of vital importance. The example of the final section shows how our theorem can be used to obtain rather complete information about the extension--information that would be difficult or impossible to obtain by any other currently known method.

\section{REFERENCES}

[1] P. M. van den Berg and J. T. Fokkema, The Rayleigh hypothesis in the theory of reflection by a grating, J. Opt. Soc. Amer. 69, 27-31 (1979)

[2] S. Christiansen and R. Kleinman, On a misconception involving point collocation and the Rayleigh hypothesis, IEEE Transactions on Antennas and Propagation 44, 1309-1316 (1996)

[3] P. Garabedian, Partial Differential Equations, Chelsea Publishing Co., New York, 1967

[4] U. Kangro and R. Nicolaides (to appear)

[5] R. F. Millar, Singularities and the Rayleigh hypothesis for solutions to the Helmholtz equation, IMA Journal of Applied Mathematics 37, 155-171 (1986)

[6] C. Morrey and L. Nirenberg, On the analyticity of the solutions of linear elliptic systems of partial differential equations, Comm. Pure Appl. Math. 10, 271-290 (1957)

[7] M. Ochmann, The source simulation technique for acoustic radiation problems, Acustica 81, 512-527 (1995)

[8] Lord Rayleigh, On the dynamical theory of gratings, Proc. Roy. Soc. London 79, 399-416 (1907) 